Como citar este artículo:

Alarcón, M. A., Alarcón, H. H., Rodríguez, L. S., Alcas, N. (2020). Intervención educativa basada en la gamificación: experiencia en el contexto universitario. Revista Eleuthera, 22(2), 117-131. DOI: 10.17151/eleu.2020.22.2.8.

\title{
Intervención educativa basada en la gamificación: experiencia en el contexto universitario*
}

\section{Gamification-based Educational intervention: experience in the university context}

\author{
Mitchell Alberto Alarcón-Diaz** \\ Henry Hugo Alarcón-Diaz ${ }^{* * *}$ \\ LISET SULAY RODRÍGUEZ-BACA**** \\ Noel AlCAS-ZAPATA*****
}

\section{Resumen}

Objetivo. Identificar la existencia de diferencias estadísticas significativas en la aplicación de la gamificación como estrategia didáctica en estudiantes universitarios de una universidad privada de Lima. Metodología. El enfoque empleado fue el cuantitativo con un diseño cuasiexperimental; el método seguido fue el hipotético-deductivo ya que a través de la contrastación de hipótesis se pudo determinar las diferencias existentes en los grupos de trabajo; participaron 72 estudiantes, 35 en el grupo control y 37 en el grupo experimental. La intervención basada en la gamificación tuvo una duración de siete semanas e incluyó actividades empleando diferentes recursos TIC. Resultados. Existen diferencias estadísticas significativas entre el grupo experimental y el grupo control; los alumnos que experimentaron a través de la gamificación muestran mejores resultados. Conclusión. Una correcta intervención pedagógica mediada por las TIC y aplique la gamificación motiva al estudiante a mejorar su aprendizaje.

Palabras clave: tecnología de la información, gestión del conocimiento, método activo, gamificación, juego educativo.

\footnotetext{
Abstract

Objective: To identify the existence of significant statistical differences in the application of gamification as a teaching strategy with university students of a private university in Lima. Methodology: The approach used was quantitative with a quasi-experimental design. The method followed was

* El presente artículo es la síntesis de un proyecto colaborativo investigativo a través del cual se intervino en una institución educativa privada de nivel superior de Lima. A través del estudio se demostró la eficacia de la inserción de los juegos virtuales en el espacio educativo mejorando el aprendizaje de los estudiantes.

*** Universidad César Vallejo, Lima, Perú. E-mail: malarcond@ucv.edu.pe

(D) orcid.org/0000-0003-0027-5701 Google Scholar

**** Universidad Nacional de Educación “Enrique Guzmán y Valle”. Lurigancho-Chosica, Perú. E-mail: halarcon@une.edu.pe (1) orcid.org/0000-0003-1588-4390 Google Scholar

***** Universidad César Vallejo. Lima, Perú. E-mail: lrodriguezb@ucv.edu.pe (D) orcid.org/0000-0003-1850-615X Google Scholar

****** Universidad César Vallejo. Lima, Perú. E-mail: nalcas@ucv.edu.pe

(D) orcid.org/0000-0001-9308-4319 Google Scholar
} 
Intervención educativa basada en la gamificación: experiencia en el contexto universitario

the hypothetical-deductive one, since through the hypothesis test it was possible to determine the differences in the work groups. A total of 72 students participated, 35 in the control group and 37 in the experimental group. The gamification-based intervention lasted 7 weeks and included activities using different ICT resources. Results: There are significant statistical differences between the experimental group and the control group. Students who experimented through gamification, show better results. Conclusion: A correct pedagogical intervention mediated by ICTs and applying gamification, motivates students to improve their learning.

Key words: information technology, knowledge management, active method, gamification, educational game.

\section{Introducción}

El último peldaño del aprendizaje formal lo constituye el espacio de la educación superior, es aquí donde se intenta profundizar en los saberes y en la autonomía de los estudiantes. Jaques Delors, en el año de 1996, le otorgó a la universidad la tarea de generar conocimientos a través de la innovación. Así, la formación integral de los estudiantes transita por la interacción entre el docente y el discente. En ese contexto, el dominio de las competencias exigidas requiere la adquisición de ciertas habilidades; la mejora del autoconcepto y la autoestima serán algunos referentes del éxito de los estudiantes (Guzmán, 2011; Hernández e Infante, 2017).

De esta forma, los procesos pedagógicos requieren de la aplicación de metodologías activas que motiven el aprendizaje y así generen conocimiento con base en trabajos colaborativos para poder solucionar problemas; ello contempla el desarrollo de nuevas habilidades que impliquen aprender y desaprender constantemente, para poder aplicar el conocimiento a diferentes situaciones (Vargas-D'uniam, Chiroque y Vega, 2016; Boude, 2017).

La necesidad de innovar se presenta en la cotidianidad de la clase, teniendo como un elemento distintivo el empleo de las TIC, las cuales hacen frente a las formas tradicionales de impartir conocimiento convirtiéndose así en una oportunidad pedagógica en la que múltiples actores interactúan y generan una motivadora dinámica grupal; así, el juego se convierte en un activador de la atención y promueve la asimilación de conceptos complejos a través de la retroalimentación inmediata, informes de progreso y recompensas (De la Hoz, Martínez, Combita y Hernández, 2019). El presente artículo tiene como propósito el presentar los resultados de una intervención cuasiexperimental en la que se empleó la estrategia de la gamificación en estudiantes de educación superior. 


\section{Referente teórico y conceptual}

\section{Reflexiones iniciales acerca de la gamificación}

La oportunidad que ofrece el aprendizaje basado en juegos es múltiple ya que las microasociaciones que se puedan crear en el aula, la libertad de vivenciar nuevas experiencias y la interacción generan motivaciones endógenas y exógenas en el estudiantado (Hernández, Monroy y Jiménez2018). A partir de diversas investigaciones (Chu \& Hung, 2016; Brull \& Finlayson, 2016) se comprobó que la gamificación es un medio para la mejora del aprendizaje, motivación y actitud de los alumnos tanto en el nivel primaria como en la preparatoria. Por otro lado, estudios como el de Alhammad \& Moreno (2018); Çakıroğlu, Başıbüyük, Güler, Atabay y Yılmaz (2017), confirmaron la eficacia de la gamificación en el nivel superior al despertar el interés hacia las asignaturas; el tino para elegir un juego, adaptarlo, contextualizarlo, aplicarlo y evaluarlo se convierten en los pasos ideales a seguir en la aplicación de esta estrategia innovadora.

Según Kapp y Zichermann y Cunningham (como se citaron en Pérez, Rivera y Delgado,2017), "con la intención de potenciar la motivación del alumnado hacia el aprendizaje, la gamificación (gamification en el ámbito anglosajón) irrumpe como una estrategia idónea para la metodología docente" (p. 114). Es decir, nos estamos refiriendo al empleo de mecánicas de juego y estrategias para generar una mayor motivación al momento de promover aprendizajes en los estudiantes.

En tal sentido, comprender la gamificación es asumir que esta se perfila hacia una actividad espontánea, a realizar en un determinado tiempo y en la que ciertas reglas definen el accionar de los estudiantes (Huizinga, 2004). En esa línea discursiva, Pascuas, Vargas y Muñoz (2017) afirman que a través de la lógica de gamificar, se podría "resolver problemas importantes de la humanidad, canalizar actitudes positivas y la colaboración en un contexto del mundo real" (p. 64). También un aspecto muy importante es que desde Vigotsky se afirmaba que la zona de desarrollo próximo es incentivada a partir de las actividades lúdicas (Moll, 1990).

Morera y Mora (2019) afirmaron que:

La gamificación como estrategia didáctica está diseñada para balancear la información de un determinado curso con jugar, y la habilidad de sus participantes de retener y aplicar la materia de este en el mundo real, en un ambiente de aprendizaje basado en tomar acciones, experimentar consecuencias y trabajar por objetivos. (p. 5) 
Intervención educativa basada en la gamificación: experiencia en el contexto universitario

De esta forma, los contenidos, la flexibilidad, la personalización y el progreso son algunos aspectos que le dan la naturaleza propia a los espacios virtuales de aprendizaje (Melo \& Díaz, 2018); al margen de los aspectos eminentemente tecnológicos, lo psicológico, por ejemplo, debería considerarse al proponer intervenciones que demanden el empleo de nuevos recursos en el espacio educativo.

\section{La gamificación dentro de las aulas}

Los estudiantes universitarios de hoy son nativos digitales; ellos crecieron y actualmente desarrollan una serie de hábitos y prácticas a través del empleo de las tecnologías; así, desarrollar competencias y habilidades en ese contexto es un reto para el quehacer docente actual. Experiencias innovadoras exitosas en las que se usaron teléfonos inteligentes, tablets, plataformas virtuales, aulas digitales y recursos informáticos diversos, demuestran el efecto de las TIC en el espacio educativo (Diez, Bañeres y Serra, 2017).

El hecho de que los estudiantes universitarios se encuentren en la etapa gamer (un momento en que los juegos de entretenimiento virtual están en su apogeo), se convierte en un ingrediente que hace propicia la aplicación de la gamificación en el contexto universitario, impulsando la resolución de situaciones complejas; la contextualización, los desafíos y las recompensas son algunas características del entorno gamer educativo (Pérez et al 2017). Estos estudiantes requieren que sus expectativas tecnológicas sean correspondidas dentro de las aulas. Ello conlleva a desarrollar iniciativas que involucren el empleo de las TIC con el fin de que el alumno se motive más y se comprometa con su propio aprendizaje (Ortiz, Jordán y Agredal, 2018).

Para Oliva (2017), una clase basada en la gamificación se orienta hacia el desarrollo del potencial humano, es "un aliciente directo que despierte en el estudiantado el interés por aprender" (p. 30). Así, el logro de los objetivos de aprendizaje se convierte en una consecuencia directa de la aplicación del juego y la acción educativa.

La medición de la gamificación transita por la consideración de una serie de dimensiones, existen propuestas que incluyen la idoneidad y pertinencia del modelo para la evaluación a realizar (Tomé, da Cunha, Farias, Rosa, Anton \& Gasparini, 2015), y por Kim \& Lee (2015); por su parte, Marache-Francisco y Brangier consideraron el aspecto moto-sensorial (como se citó en De Puy y Miguelena, 2017).

En este estudio, la medición de la variable gamificación consideró aquellos elementos que sugieren una coherencia entre sí, desde los aspectos generales hasta los específicos; en tal sentido se considerarán: lo dinámico (la curiosidad, lo narrativo, experiencia, reglas, relaciones y sorpresas), lo mecánico (el involucramiento del jugador a través de la creación, exploración, 
estado de éxito y recolección de información) y los componentes (los recursos disponibles tales como niveles, clasificaciones, misiones, avatares y preguntas) (García e Hijón, 2017).

\section{Metodología}

El enfoque de este estudio es cuantitativo, con diseño cuasiexperimental y longitudinal, cuyo propósito fue demostrar que la intervención educativa basada en la gamificación de los estudiantes de una universidad privada de Lima mejora su proceso de interacción. Se incluyó una muestra de 72 estudiantes universitarios de una misma escuela, distribuidos en dos grupos: control de 35 estudiantes y el grupo experimental con 37 estudiantes. Durante la experimentación, la organización de las actividades propuestas se dirigió a establecer una lógica que correspondía con los principios propuestos por la teoría de la gamificación. Así, la asignación de puntajes a cada actividad reveló como de manera progresiva los estudiantes se iban comprometiendo cada vez más con la clase. Por otro lado, los grados de dificultad propuestos inicialmente fueron superados por los estudiantes demostrándose así el avance respectivo, cada fase superada implicó un premio o un regalo otorgado; se fueron clasificando a los estudiantes con base en el logro de objetivos, configurando finalmente un ranking en el aula. Los retos o desafíos también fueron parte de la intervención, de manera individual y grupal se desarrollaron actividades desafiantes que se relacionaron con la solución a problemas sugeridos.

Para el caso de la recolección de datos, la técnica utilizada en este estudio fue la encuesta; se elaboró un cuestionario con 15 ítems con escala ordinal tipo Likert de cinco opciones de respuesta y para las siguientes dimensiones: dinámica (5 ítems), mecánica (5 ítems) y componentes ( 5 ítems). Para verificar si el instrumento era confiable, se utilizó el estadístico Alfa de Cronbach a una muestra de estudiantes que no participaron en este estudio y que tuvieron las mismas características de los sujetos que fueron parte de la experiencia; como resultado se obtuvo el valor de 0,891 , lo cual demuestra que el instrumento utilizado era confiable.

Para el análisis estadístico de los datos se aplicó inicialmente la estadística descriptiva a cada una de las dimensiones y a toda la variable gamificación, generándose figuras piramidales con su correspondiente descripción y análisis. Asimismo, en los resultados inferenciales inherentes a las pruebas de hipótesis, se decidió utilizar el estadístico U de Mann-Whitney, para demostrar la existencia de diferencias significativas entre los grupos de control y experimental, para la variable gamificación y sus correspondientes dimensiones. 


\section{Resultados}

\section{Resultados descriptivos}

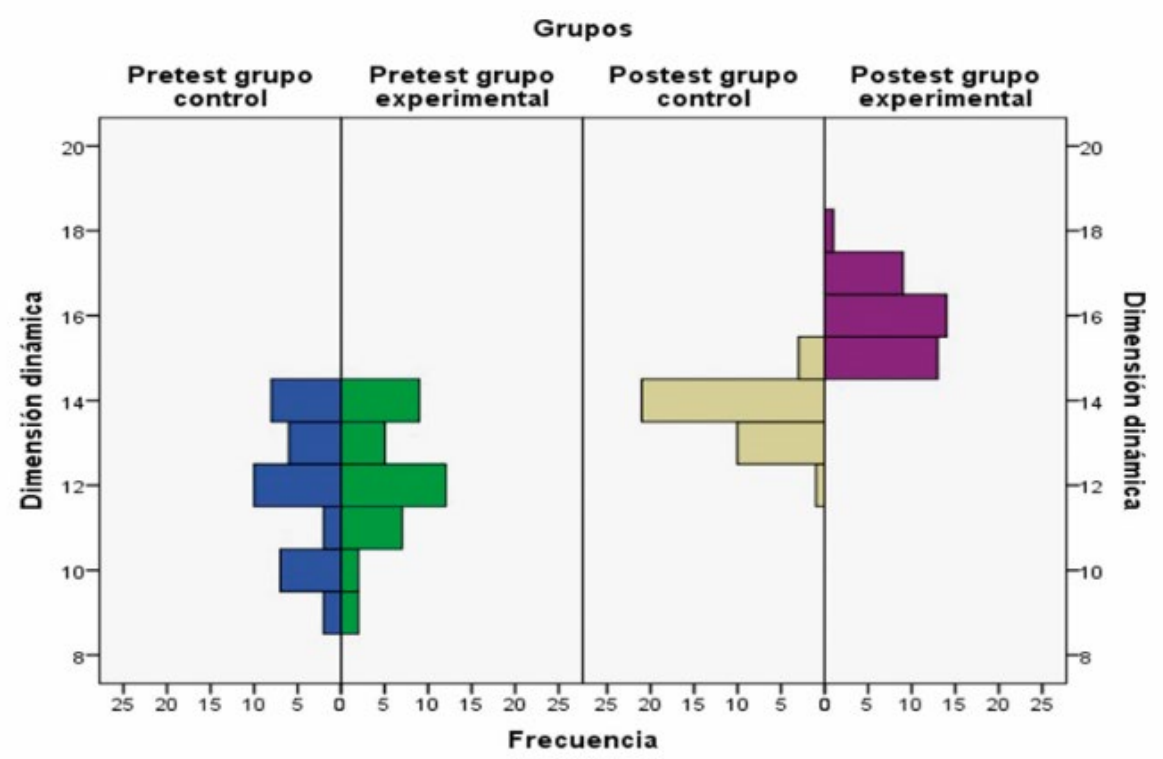

Figura 1. Dimensión dinámica: resultados del pretest y postest de los grupos

de control y experimental.

Fuente: Software SPSS

En la figura 1 se observan los resultados de la interacción de los estudiantes, según el pretest y postest de los grupos de control y experimental. En este diagrama de pirámide se evidencia además en la situación inicial de ambos grupos (pretest), la mediana es igual. Por otra parte, en la situación final tanto en el grupo de control como experimental (postest), la mediana de las interacciones de los estudiantes en la dimensión dinámica del grupo experimental es mayor que la mediana del grupo de control. Situación por la cual, se puede asumir que esta mejora en la interacción de los estudiantes universitarios en la dimensión dinámica, se origina por el proceso de experimentación. 


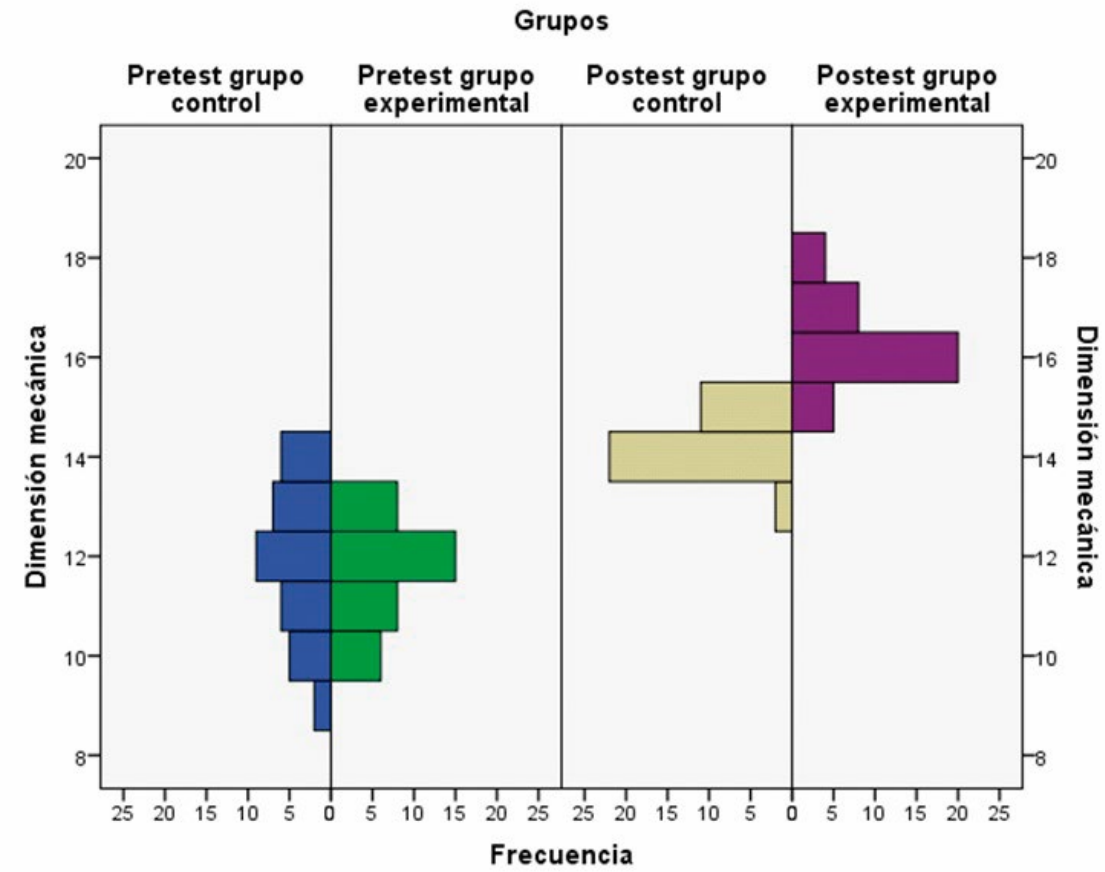

Figura 2. Dimensión mecánica: resultados del pretest y postest de los grupos de control y experimental.

Fuente: Software SPSS

En la figura 2 se observan los resultados de la interacción de los estudiantes en la dimensión mecánica, según el pretest y postest de los grupos de control y experimental. En este diagrama de pirámide se muestra además en la situación inicial de ambos grupos (pretest), un estado casi similar. Por otra parte, en la situación final, tanto en el grupo de control como experimental (postest), la mediana de las interacciones de los estudiantes en la dimensión mecánica del grupo experimental es mayor que la mediana del grupo de control. Situación por la cual se puede asumir que esta mejora en la interacción de los estudiantes universitarios en la dimensión mecánica se origina por el proceso de experimentación. 


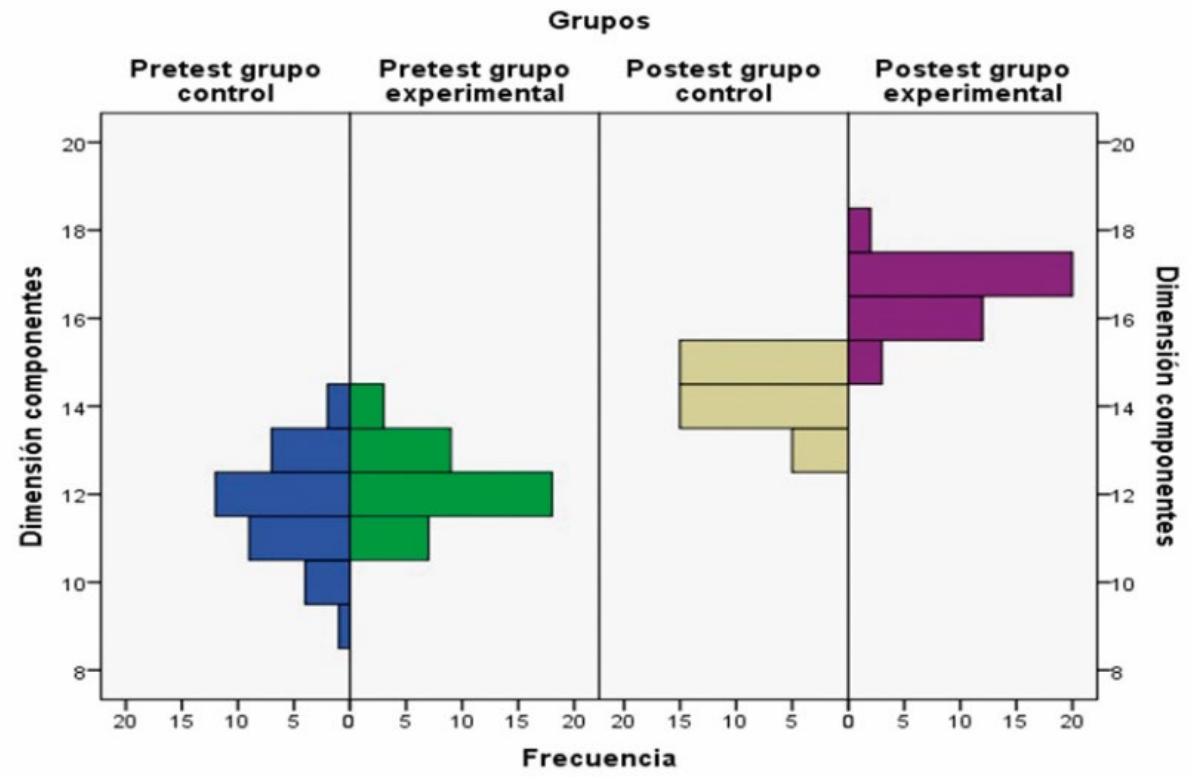

Figura 3. Dimensión componentes: resultados del pretest y postest de los grupos de control y experimental.

Fuente: Software SPSS

En la figura 3 se presentan los resultados de la interacción de los estudiantes en la dimensión componentes, según el pretest y postest de los grupos de control y experimental. En este diagrama de pirámide se muestra además en la situación inicial de ambos grupos (pretest), la mediana es igual. Por otra parte, en la situación final, tanto en el grupo de control como experimental (postest), la mediana de las interacciones de los estudiantes en la dimensión componentes del grupo experimental es mayor que la mediana del grupo de control. Situación por la cual, se puede asumir que esta mejora en la interacción de los estudiantes universitarios en la dimensión componentes se origina por el proceso de experimentación. 


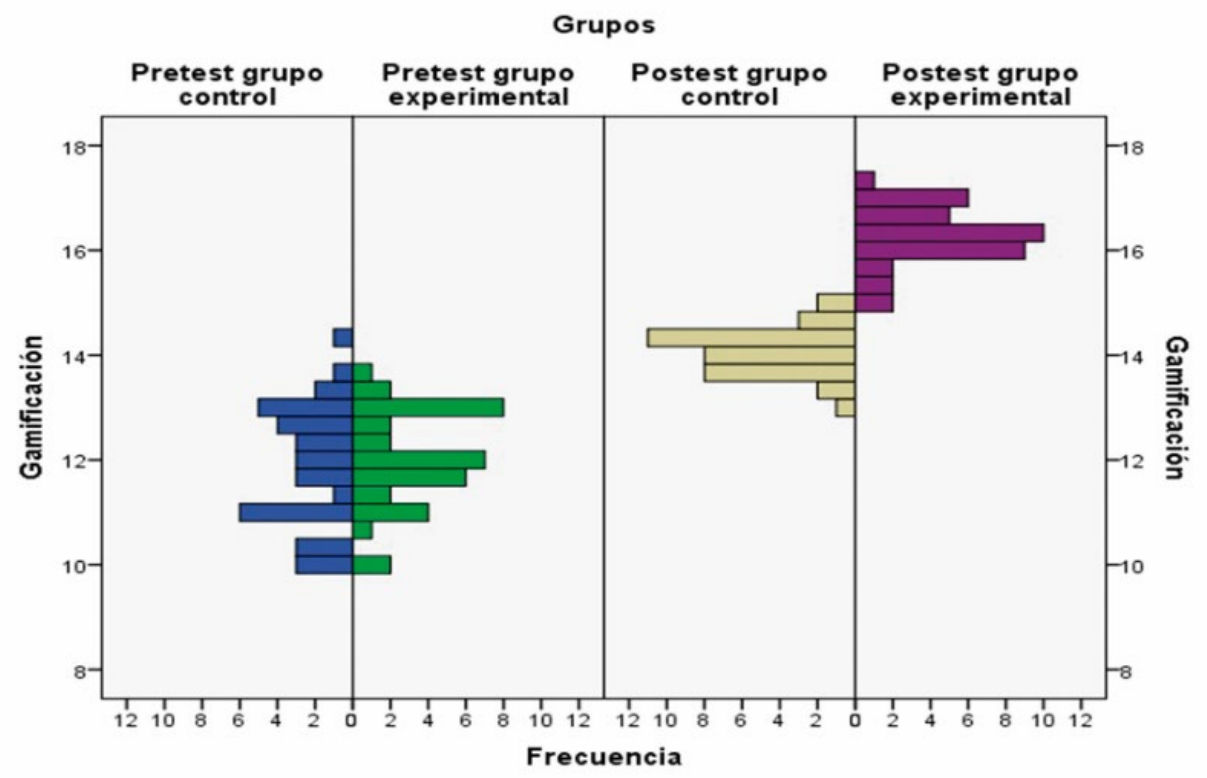

Figura 4. Gamificación: resultados del pretest y postest de los grupos de control y experimental.

Fuente: Software SPSS

En la figura 4 se presentan los resultados de la interacción de los estudiantes en la variable gamificación, según el pretest y postest de los grupos de control y experimental. En este diagrama de pirámide, se muestra además que en la situación inicial de ambos grupos (pretest), la mediana es casi similar. Asimismo, en la situación final, tanto en el grupo de control como experimental (postest), la mediana de las interacciones de los estudiantes en la gamificación del grupo experimental es mayor que la mediana del grupo de control. Situación por la cual se puede asumir que esta mejora en la interacción de los estudiantes universitarios en la gamificación se origina por el proceso de experimentación. 
Intervención educativa basada en la gamificación: experiencia en el contexto universitario

\section{Resultados inferenciales}

Tabla 1. Rangos de la variable gamificación.

\begin{tabular}{lllll}
\hline & Grupos & N & $\begin{array}{l}\text { Rango } \\
\text { promedio }\end{array}$ & $\begin{array}{l}\text { Suma de } \\
\text { rangos }\end{array}$ \\
\hline Gamificación & Postest grupo control & 35 & 18,06 & 632,00 \\
& Postest grupo experimental & 37 & 53,95 & 1996,00 \\
& Total & 72 & & \\
\hline
\end{tabular}

Fuente: Software SPSS

En la tabla 1, la diferencia de los rangos del postest entre los grupos de control y experimental evidencia que existe una diferencia significativa.

Tabla 2. Estadístico de prueba U de Mann-Whitney.

\begin{tabular}{ll}
\hline & Gamificación \\
\hline U de Mann-Whitney & 2,000 \\
W de Wilcoxon & 632,000 \\
Z & $-7,317$ \\
Sig. asintótica (bilateral) &, 000 \\
\hline
\end{tabular}

a. Variable de agrupación: Grupos

Fuente: Software SPSS

En la tabla 2, se observa que el estadístico U de Mann-Whitney es igual a 632,000, con z = $-7,317$ y una significancia empírica asintótica (bilateral) $=0,000<0,05$, lo cual indica que existen diferencias significativas en el pretest y postest de la gamificación entre los grupos de control y experimental.

Tabla 3. Rangos de las dimensiones de la variable gamificación.

\begin{tabular}{llccc}
\hline Dimensiones & Grupos & N & Rango promedio & $\begin{array}{l}\text { Suma de } \\
\text { rangos }\end{array}$ \\
\hline \multirow{3}{*}{ Dinámica } & Postest grupo control & 35 & 18,56 & 649,50 \\
& Postest grupo experimental & 37 & 53,47 & 1978,50 \\
& Total & 72 & & \\
Mecánica & Postest grupo control & 35 & 18,79 & 657,50 \\
& Postest grupo experimental & 37 & 53,26 & 1970,50 \\
& Total & 72 & & \\
& Postest grupo control & 35 & 18,79 & 657,50 \\
& Postest grupo experimental & 37 & 53,26 & 1970,50 \\
& Total & 72 & & \\
\hline
\end{tabular}

Fuente: Software SPSS 
La tabla 3 muestra los resultados de los rangos promedios del estadístico U de Mann-Whitney. Se observa, además, que la diferencia entre los rangos promedio de la dimensión dinámica entre los grupos de control y experimental es de 34,91. Asimismo, en las dimensiones mecánica y componentes, esta diferencia de rangos promedio entre ambos grupos es similar y equivale a 34,47 .

Tabla 4. Estadísticos de prueba de las dimensiones de la gamificación.

\begin{tabular}{llll}
\hline & Dimensión dinámica & $\begin{array}{l}\text { D i m e n s i ón } \\
\text { mecánica }\end{array}$ & $\begin{array}{l}\text { D i m e n s i ó n } \\
\text { componentes }\end{array}$ \\
\hline U de Mann-Whitney & 19,500 & 27,500 & 22,500 \\
W de Wilcoxon & 649,500 & 657,500 & 652,500 \\
Z & $-7,250$ & $-7,214$ & $-7,228$ \\
Sig. asintótica (bilateral) &, 000 &, 000 &, 000 \\
\hline
\end{tabular}

a. Variable de agrupación: Grupos

Fuente: Software SPSS

La tabla 4 muestra los resultados de la prueba estadística para las hipótesis específicas de cada una de las dimensiones de la gamificación: dimensión dinámica (U de Mann-Whitney $=19,500 ; \mathrm{z}=-7,250$; sig. asintótica bilateral $=, 000<0,05)$, dimensión mecánica (U de Mann-Whitney $=27,500 ; \mathrm{z}=-7,214$; sig. asintótica bilateral $=, 000<0,05)$ y dimensión componentes (U de Mann-Whitney $=22,500 ; \mathrm{z}=-7,228$; sig. asintótica bilateral $=, 000<$ $0,05)$. Esto indica, además, que existen diferencias significativas en el pretest y postest en las dimensiones: dinámica, mecánica y componentes, entre los grupos de control y experimental.

\section{Discusión}

En este estudio se comprueba que el incorporar la gamificación como estrategia en el aula determina diferencias significativas en relación con aquellos contextos que realizan su quehacer de manera tradicional. Los resultados de la estadística descriptiva muestran que los valores del grupo experimental fueron mejores que los del grupo control en cada una de las dimensiones propuestas: dinámica, mecánica y componentes; desde la teoría del aprender haciendo propuesto por Dewey hasta la reacción emocional sostenida por Kapp, las condiciones de aprendizaje y los estímulos por aprender se favorecen al culminar la experimentación. Esto refuerza la premisa la cual sostiene que las innovaciones acertadas conducen a resultados exitosos.

Investigaciones como las de Quintanal (2016) y Godoy (2019) enfocan el éxito de sus experiencias gamificadoras a partir de la mejora de los niveles de rendimiento académico logrado. También se evidencia una mejora de los niveles de autonomía, autoconfianza y 
Intervención educativa basada en la gamificación: experiencia en el contexto universitario

autoestima. A partir de ello, se entiende que la educación de hoy se orienta a una actividad más dinámica, constructiva (en la que el estudiante es el centro del proceso) y en el que las tecnologías cumplen un rol preponderante. Los resultados inferenciales confirman la diferencia estadística significativa de la aplicación de la gamificación del grupo experimental por sobre el grupo control; no se evidencia una marcada diferencia de una dimensión sobre las otras, se asume que por ser la primera vez en la que los estudiantes participaron de esta estrategia, los aspectos tales como la curiosidad, el interés por involucrarse en las actividades y la habilidad al momento de usar los diferentes recursos (es decir lo dinámico, lo mecánico y los componentes) se pusieron en práctica de manera uniforme por parte de los estudiantes.

A partir de los resultados, se corrobora la tesis teórica de que el empleo de estrategias novedosas puede lograr una mejor apropiación del saber (Núñez, Gaviria, Tobón, Guzmán y Herrera, 2019); la sola presencia del recurso TIC en las aulas no asegura un mejor aprendizaje, es necesario modificar la dinámica del docente y del estudiante (Miralles, Gómez y Monteagudo, 2019).

\section{Conclusiones}

La práctica docente dentro de las aulas está pasando por una constante transformación. La demanda de los estudiantes es cada vez mayor; se exige ser más creativo, innovador, aportar soluciones y reconfigurar la realidad. La gamificación como estrategia suma a la acción docente el valor de los juegos, la planificación a través de acciones predefinidas, el generar una mayor motivación y el involucramiento de los estudiantes.

En este estudio se determinó que la intervención educativa innovadora basada en la gamificación mejoró la interacción de los estudiantes de una universidad privada de Lima; esto se demuestra con la existencia de diferencias significativas en el pretest y postest de la variable gamificación, entre los grupos de control y experimental, según el estadístico: U de Mann-Whitney = 632,$000 ; \mathrm{z}=-7,317 ; \mathrm{y}$ sig. asintótica bilateral $=, 000<0,05$.

\section{Referencias}

Alhammad, M. \& Moreno, A. (2018). Gamification in software engineering education: A systematic mapping. Journal of Systems of Software, 141, 131-150. doi: 10.1016/J. JSS.2018.03.065.

Boude, O. (2017). Estrategias de aprendizaje para formar en Educación Superior a una generación interactiva. Educación Médica Superior, 31(2). Recuperado de http: / / scielo.sld. $\mathrm{cu} /$ scielo.php?script $={ }_{\text {sci_arttext\&pid }}=$ S0864-21412017000200012\&lng $=$ es\&tlng $=$ es. 
Brull, S. \& Finlayson, S. (2016). Importance of Gamification in Increasing Learning, J. Contin. The Journal of Continuing Education in Nursing, 47(8), 372-375. doi: 10.3928/0022012420160715-09.

Çakıroğlu, Ü., Başıbüyük, B., Güler, M., Atabay, M. \& Yılmaz, B. (2017). Gamifying an ICT course: Influences on engagement and academic performance. Computers in Human Behavior, 69. doi: 10.1016/J.CHB.2016.12.018.

Chu, C. \& Hung, C. (2015). Effects of the Digital Game-Development Approach on Elementary School Students' Learning Motivation, Problem Solving, and Learning Achievement. International Journal of Distance Education Technologies (IJDET), 13(1), 87-102. doi: 10.4018/ ijdet. 2015010105.

De la Hoz, E., Martínez, O., Cómbita, H. y Hernández, H. (2019). Las Tecnologías de la Información y la Comunicación y su Influencia en la Transformación de la Educación Superior en Colombia para Impulso de la Economía Global. Información Tecnológica, 30(1), 255-262. doi: https: / /dx.doi.org/10.4067/S0718-07642019000100255.

De Puy, M. y Miguelena, R. (2017). Importancia de la Gamificación en la Educación Aplicado en Entornos de la Investigación. Recuperado de http://www.laccei.org/LACCEI2017BocaRaton/student_Papers/SP282.pdf.

Diez, J., Bañeres, D. y Serra, M. (2017). Experiencia de gamificación en secundaria en el aprendizaje de sistemas digitales. Education in the knowledge Society, 18(2), 85-105. doi: https:/ / doi.org/10.14201/eks201718285105.

García, M. e Hijón, R. (2017). Análisis para la gamificación de un curso de Formación Profesional. IE Comunicaciones: Revista Iberoamericana de Informática Educativa, 26, 46-60. Recuperado de https: / / dialnet.unirioja.es/servlet/articulo?codigo=6231883.

Godoy, M. (2019). La Gamificación desde una Reflexión Teórica como recurso estratégico en la Educación. Revista Espacio, 40(15), 25. Recuperado de http://www.revistaespacios. com/a19v40n15/19401525.html.

Guzmán, J. (2011). La calidad de la enseñanza en educación superior ¿Qué es una buena enseñanza en este nivel educativo? Perfiles Educativos, 33(spe), 129-141. Recuperado de http: / / www. scielo.org.mx/scielo.php?script $=$ sci_arttext\&pid $=$ S0185-26982011000500012\&lng $=\mathrm{es}$ $\& \operatorname{tng}=\mathrm{es}$.

Hernández, I., Monroy, A. y Jiménez, M. (2018). Aprendizaje mediante Juegos basados en Principios de Gamificación en Instituciones de Educación Superior. Formación Universitaria, 11(5), 31-40. doi: http:/ /dx.doi.org/10.4067/S0718-50062018000500031.

Hernández, R. e Infante, M. (2017). La clase en la educación superior, forma organizativa esencial en el proceso de enseñanza-aprendizaje. Educación y Educadores, 20(1), 27-40. doi: http:/ /dx.doi.org/10.5294/edu.2017.20.1.2. 
Intervención educativa basada en la gamificación: experiencia en el contexto universitario

Huizinga, J. (2004). Homo ludens. Madrid, España: Alianza Editorial.

Kim, J. \& Lee, W. (2015). Dynamical model for gamification of learning (DMGL). Multimedia Tools and Applications, 74(19). Recuperado de https: / /link. springer.com/article/10.1007/ s11042-013-1612-8.

Melo, D. \& Díaz, P. (2018). Emotional Learning and Gamification in Virtual Education Environments. Información Tecnológica, 29(3), 237-248. doi: https: / / dx.doi.org/10.4067/ S0718-07642018000300237.

Miralles, P., Gómez, C. y Monteagudo, J. (2019). Percepciones sobre el uso de recursos TIC y "MASS-MEDIA" Para la enseñanza de la historia. Un estudio comparativo en futuros docentes de España-Inglaterra. Educación XX1, 22(2). doi: https://doi.org/10.5944/ educxx1.21377.

Moll, L. (1990). La zona de desarrollo próximo de Vygotski: una reconsideración de sus implicaciones para Ia enseñanza. Infancia y Aprendizaje, 13(51-52), 247-254. doi: https:/ / doi.org/10.1080/02103702.1990.10822280.

Morera, J. y Mora, J. (2019). Use of Gamification in Fundamentals of Biology Course. Revista Electrónica Educare, 23(2), 1-13. doi: https: / / doi.org/10.15359/ree.23-2.10.

Núñez, C., Gaviria, J., Tobón, S., Guzmán, C. y Herrera, S. (2019). La práctica docente mediada por TIC: una construcción de significados. Revista Espacios, 40(5), 4. Recuperado de https: / / revistaespacios.com/a19v40n05/a19v40n05p04.pdf.

Oliva, H. (2017). La gamificación como estrategia metodológica en el contexto educativo universitario. Realidad y Reflexión, 44, 29-47. doi: https://doi.org/10.5377/ryr. v44i0.3563.

Ortiz, A., Jordán, J. y Agredal, M. (2018). Gamificación en educación: una panorámica sobre el estado de la cuestión. Educação e Pesquisa, 44, e173773. doi: https://dx.doi. org/10.1590/s1678-4634201844173773.

Pascuas, Y., Vargas, E. y Muñoz, J. (2017). Experiencias motivacionales gamificadas: una revisión sistemática de literatura. Innovación Educativa, 17(75), 63-80. Recuperado de http:/ / www.scielo.org.mx/pdf/ie/v17n75/1665-2673-ie-17-75-63.pdf.

Pérez, I., Rivera, E. y Delgado, M. (2017). Mejora de hábitos de vida saludables en alumnos universitarios mediante una propuesta de gamificación. Nutrición Hospitalaria, 34(4), 942 951. doi: http://dx.doi.org/10.20960/nh.669.

Quintanal, F. (2016). Aplicación de herramientas de gamificación en física y química de secundaria. Opción: Revista de Ciencias Humanas y Sociales, 32(12). 327-348. Recuperado de https: / / dialnet.unirioja.es/servlet/articulo?codigo $=5852309$. 
Tomé, A., Da Cunha, L., Farias, M., Rosa, B., Anton, A. \& Gasparini, I. (2015). Gamification in e-Learning Systems: A Conceptual Model to Engage Students and Its Application in an Adaptive e-Learning System. In P. Zaphiris. \& A. Ioannou. (Ed.), Learning and Colaboration Technologies (pp. 595-607). Cham, Suiza: Springer International Publishing.

Vargas-D'uniam, J., Chiroque, E. y Vega, M. (2016). Innovación en la docencia universitaria: una propuesta de trabajo interdisciplinario y colaborativo en educación superior. Educación, 25(48), 67-84. doi: https://dx.doi.org/10.18800/ educacion.201601.004. 